\title{
Contaminación de suelos con huevos de Toxocara sp. en ambientes internos de Instituciones Educativas Estatales de los distritos del cono Norte de Lima.
}

Soil contamination with Toxocara eggs in indoor environments of Educational Instutions from North Lima.

Jackeline Ramírez ${ }^{1}$, Néstor Falcón ${ }^{1}$, Enrique Serrano $^{1}$

\section{RESUMEN}

Objetivo:Determinar la presencia de huevos de T. canis en el suelo de Instituciones Educativas del Norte de Lima. Metodología: Se recolectó una muestra de suelo en 30 instituciones estatales educativas (IEE)de los siguientes distritos de Lima norte: Comas ( $\mathrm{n}=17)$, Puente Piedra $(\mathrm{n}=8)$, Ancón $(\mathrm{n}=3)$ y Carabayllo $(\mathrm{n}=2)$. Todas las muestras se procesaron mediante la técnica de concentración por flotación. Resultados y Conclusiones: Se obtuvieron dos muestras positivas lo que representa una frecuencia $6.7 \%$. Las IEE que resultaron positivas correspondían al distrito de Comas y Puente Piedra.

Palabras clave: Toxocariasis, zoonosis parasitaria, Lima norte.

\section{ABSTRACT}

Aim: To determine the presence of Toxocara canis eggs on the ground of some Public Schools in North Lima. Methods: Thirty ground samples were collected from Public Schools in the following North Lima districts: Comas $(\mathrm{n}=17)$, Puente Piedra ( $\mathrm{n}=8)$, Ancón $(\mathrm{n}=3)$ and Carabayllo $(\mathrm{n}=2)$. Samples were processed using the Concentration by Flotation Technique. Results: Two samples positive, which represents a frequency of $6.7 \%$. The positive samples belong to to the districts of Comas and Puente Piedra.

Key words: Toxocariasis, zoonosis, Toxocara eggs, North Lima.

\section{INTRODUCCIÓN}

Según el Comité de Expertos en Zoonosis Parasitarias la toxocariasis es una de las parasitosis consideradas como un problema serio para la salud pública (Miranda-Sousa et al., 1999). La ingestión accidental de huevos larvados con el segundo estadio de Toxocara sp. produce en el hombre y en especial al grupo de riesgo (niños, gestantes, pacientes geriátricos $\mathrm{y}$ personas que tienen el perfil inmunológico deprimido) diversos síndromes entre los que las formas de larva migrante visceral (LMV) y larva migrante ocular (LMO) son los más comunes (Atías et al., 1994; Leguía et al, 2002).

Estudios de seroprevalencia de toxocariasis en personas en el Perú han encontrado $27.9 \%$ en el distrito Perené (Espinoza et al, 2006), 32.4\% en niños del distrito de Mórrope (Espinoza et al, 2008) y 46.7\% en niños de instituciones educativas en el distrito de San Juan de Lurigancho (Breña et al., 2007). 
Los factores epidemiológicos asociados con la exposición a Toxocara sp. son la escases de agua potable, contacto con el suelo, ausencia de alcantarillado y tenencia de mascotas (Mendoza et al., 2010). La diseminación de la materia fecal es una de las causas que contribuyen a infección humana ya que a ella tienen acceso las personas en forma accidental directa o indirectamente (Hendrix et al., 1996).

Estudios en Argentina han encontrado que personas que no convivían con mascotas, se encontraban infectadas con el parasito, lo que ha llevado a considerar la importancia de la contaminación con materia fecal canina en áreas de recreación pública, lugares de juego de niños y calles de la ciudad (Minvielle et al., 2003). El gran número de perros callejeros, con o sin dueño, presentes en las calles de la ciudad, asociados al libre ingreso de zonas recreacionales, aumenta el riesgo de infección en los niños (Scaini et al., 2003). En el Perú, se ha encontrado parques públicos de Lima Metropolitana y Callao contaminados con huevos de Toxocara sp., reportándose prevalencias de $34 \pm 9 \%, 30 \pm 9 \%, 41 \pm$ $8 \%, 63 \pm 9 \%$ y $37 \pm 11 \%$, para los distritos de los conos norte, sur, este, oeste y el Callao, respectivamente. La evaluación de parques clasificados según su ubicación en los cinco estratos socioeconómicos demostró que había mayor contaminación en los parques que se encuentran ubicados en los estratos socioeconómicos altos y medio altos (Chávez et al., 2002).

Un estudio reciente a escolares de ocho Instituciones Educativas Estatales (IEE) del cono norte, encontró que el $27.6 \%$ de los parques a los que acudían los escolares, se encontraban contaminados con huevos de $T$. canis. También se encontró huevos de este parásito en suelos en dos de siete colegios muestreados $(28.6 \%)$ y en 11 de 120 muestras suelos de hogares (9.2\%); (Guillinta, 2011).

Los estudios de contaminación ambiental con huevos de $T$. canis han sido dirigidos principalmente a lugares de recreación de los niños (parques). Sin embargo, el ambiente del colegio es otro lugar en donde estos permanecen un importante tiempo del día, por lo que se hace necesario investigar dichos ambientes toda vez que la mayoría de los colegios poseen perros guardianes o a ellos tiene acceso los perros que acompañan a los niños cuando estos son llevados al colegio. Estos animales deambulan libremente y defecan en el ambiente, lo que puede producir una contaminación del mismo con huevos o parásitos zoonoticos si es que no se encuentran debidamente desparasitados. Por ello, el objetivo del estudio fue determinar la presencia de huevos de
Toxocara sp en muestras de suelo de Instituciones Educativas Estatales del Cono Norte de Lima.

\section{MATERIALES Y METODOS}

El estudio de investigación de corte transversal y descriptivo, tipo prevalencia, se realizó en Instituciones Educativas Estatales (IEE) de los distritos de Comas, Carabayllo, Puente Piedra, Santa Rosa y Ancón. Las fechas de recojo de muestras se distribuyeron entre los meses de octubre del 2012 hasta febrero del 2013. Se estudiaron 30 IEE con población estudiantil de nivel primario mayor a 500 niños. El diagnóstico parasitológico se realizó en el Laboratorio de Parasitología de la Facultad de Medicina Veterinaria y Zootecnia de la Universidad Peruana Cayetano Heredia. FAVEZ-UPCH.

El estudio se llevó a cabo con previa autorización del director de cada IEE. Asimismo, se les hizo entrega de los resultados de la evaluación de los suelos de sus ambientes y las recomendaciones acerca del manejo sanitario de los mismos. Los resultados del diagnóstico parasitológico de las IEE participantes fueron manejados de manera confidencial.

Se recolectaron muestras de suelos y jardines de cada IEE la misma que era reunida en un solo envase, identificándose como una única muestra. Las muestras de jardines y suelos arenosos se obtuvieron a través de la metodología de las dos "W". El método consistió en trazar en el área a muestrear dos "W" opuestas entre sí, luego se determinó la longitud en pasos de cada "W y por cada $10 \%$ de la extensión de cada "W" se colectaron porciones de suelo. De cada una de estas partes se colectó cuatro pequeñas cantidades de suelo, llegándose a obtener entre 1000 y 3000 g de cantidad colectada en el total de las áreas (jardín y suelos arenosos). La recolección de la muestra de los patios se realizó a través de un barrido de los suelos de los colegios participantes. De ellos se consideró un peso de 1000 a $2000 \mathrm{~g}$.

Las muestras de suelos de cada colegio fueron evaluadas mediante la técnica de concentración por flotación con solución sobresaturada de $\mathrm{NaCl}$, considerándose positiva aquella muestra que presentaba al menos un huevo de Toxocara sp.

El análisis de datos incluyó el cálculo de la frecuencia de muestras positivas para el total de IEE involucradas en el estudio y para las IEE de cada distrito. 


\section{RESULTADOS}

La distribución de las IEE participantes en el estudio fue la siguiente: tres del distrito de Ancón, dos de Carabayllo, diecisiete de Comas y ocho de Puente Piedra.

La figura 1 muestra la imagen fotográfica de los huevos de $T$. canis diagnosticados microscópicamente en el Laboratorio de Parasitología de la FAVEZ - UPCH.

Del total de muestras recolectadas, dos resultaron positivas lo que correspondió a una frecuencia relativa del $6.7 \%$. Las muestras positivas correspondieron a una IEE del distrito de Puente Piedra y una de Comas (ver cuadro 1)

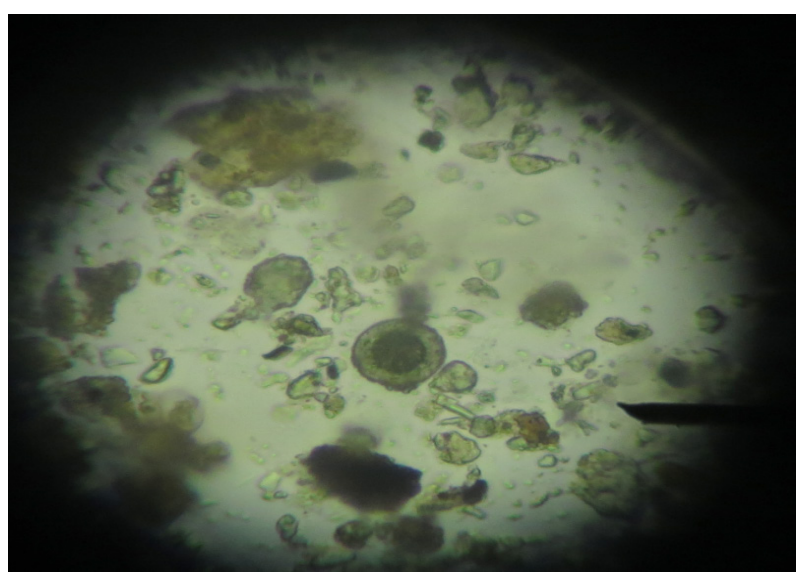

Figura 1. Huevo de T. canis obtenido de la muestra de ambiente de la IEE del distrito de Comas (40X).

Cuadro 1. Muestras de suelos positivos a T. canis en ambientes de Instituciones Educativas Estatales de distritos del cono norte de Lima.

\begin{tabular}{lccc}
\hline Distrito & Total de & \multicolumn{2}{c}{ Suelos de IEE } \\
puelos de IEE & \multicolumn{2}{c}{ positivas } \\
evaluados & Nro. & $\%$ \\
\hline Ancón & 3 & 0 & 0 \\
Carabayllo & 2 & 0 & 0 \\
Comas & 17 & 1 & 5.9 \\
Puente Piedra & 8 & 1 & 12.5 \\
TOTAL & 30 & 2 & 6.7 \\
\hline
\end{tabular}

\section{DISCUSIÓN}

Una serie de estudios realizados en parques públicos, han evaluado la presencia de huevos de $T$. canis y determinado el riesgo que representa para la salud pública (Chávez et al., 2002). Sin embargo, un primer estudio realizado por Guillinta (2011) encontró huevos de $T$. canis en ambientes en dos de siete colegios del cono norte de Lima. El hallazgo de ambientes contaminados en dos IE (2/30) sugiere que en estos lugares los niños pueden estar expuestos a huevos de Toxocara sp. Los huevos del parásito se encontraron en áreas donde los niños permanecen la mayor cantidad de tiempo cuando están fuera de las aulas de clase. En estos espacios los niños juegan y comen, y en consecuencia podrían estar expuestos a contraer la toxocaríasis.

Las probables fuentes de contaminación pueden estar en los perros guardianes que suelen ser soltados en la noche para cumplir su función de vigilancia y que en otros casos se encuentran libres todo el día, perros que acompañan a los niños y cuyo ingreso al colegio no se restringe, y perros callejeros que se acercan al colegio con la finalidad de buscar comida. Estos animales deambulan libremente en los diferentes ambientes de las IEE y defecan en zonas donde los niños juegan o comen. Considerando que la mayoría de ellos no cumplen con un programa sanitario adecuado (no se les desparasita periódicamente), es que se sospecha que estos animales estarían actuando como reservorio y diseminadores de huevos de diferentes parásitos.

Otra forma de contaminación puede ser de tipo mecánico. En este caso, las personas juegan un papel importante en la diseminación de huevos de parásitos, ya que estos pueden ser trasladados en los zapatos, tanto desde la acera, parques públicos o casas que pueden estar contaminados con huevos de Toxocara $\mathrm{sp}$.

Las IEE que resultaron positivas estuvieron ubicadas en los distritos de Puente Piedra y Comas. El colegio de Comas no contaba con perros guardianes y el de Puente Piedra sí. Estos resultados sugieren que independientemente de la tenencia de perros, los ambientes pueden ser contaminados por perros ajenos a la IEE o por medios mecánicos, pudiendo estos representar un foco de infección para sus propios estudiantes.

La presencia de huevos de T. canis dentro de los ambientes internos de las IEE es preocupante debido a que estos pueden sobrevivir, a temperatura y humedad adecuada, alrededor de tres años en los suelos, lo que representa un potencial riesgo de infección para los estudiantes (Delgado et al., 2009; Huapaya et al., 2009; Armstrong et al., 2011). 
Debido a que los niños son una población vulnerable se debería tomar precauciones para que disminuya el riesgo de infección por Toxocara sp. Para ello, se debe llevar un buen control de desparasitación de los perros guardianes de las IEE, evitar el ingreso de perros callejeros ajenos a los centros educativos. Es evidente que el control de la transmisión mecánica es compleja, sin embargo se debe de tener cuidado para no pisar heces que se encuentran dispersas en las calles y trasladarlos. La promoción de los hábitos de higiene como el lavado de manos después de jugar con las mascotas y antes de consumir alimentos debe de ser reforzado a partir de los hogares e IEE.

Los docentes de los colegios deben de concientizar a padres de familia, niños y a sus colegas acerca de la importancia de dar a conocer la capacidad zoonótica que tienen estos parásitos y otros de interés en salud pública y así evitar que los niños se expongan a contraer esta enfermedad. Estos conocimientos deben de incluir la forma de transmisión de los parásitos, las consecuencias que producen sobre la salud de las personas y la forma como prevenir su contagio.

El personal de los centros educativos tanto director como profesores deben de ser asesorados por un Médico Veterinario con el fin de que se evalúe a los animales guardianes e iniciar en ellos un programa sanitario con el fin de cortar el ciclo biológico de los parásitos. También se debe de capacitar al personal de limpieza para que recoja, manipule y elimine de manera adecuada las heces de los canes. Los lavaderos de los centros educativos deben de contener jabón desinfectante a la mano y se debe brindar la concientización de los niños para que hagan el uso correcto de ellos y así puedan estar protegidos. Asimismo, los perros guardianes de los centros educativos deberían estar encerrados cuando los niños jueguen o coman en su horario de refrigerio para evitar que ello acaricien o besen a los perros o que estos laman a los niños, teniendo en cuenta que estos animales tienen la costumbre de lamerse la zona perianal de donde puede trasladar huevos de parásitos o parásitos adultos y así disminuir el riesgo de infección en los niños.

A fin de mejorar los conocimientos referentes a temas de salud se debe de capacitar a los docentes o tutores de los estudiantes en estos temas y estos a su vez hacer la réplica a los estudiantes. La información ofrecida por sus propios profesores tiene una mayor probabilidad ser adoptados e internalizados por los estudiantes. Además, una vez que ellos lo adoptan es probable que se produzcan un efecto multiplicador y ello se debería de aprovechar para hacer llegar los mismos a sus padres. Se espera que estos esfuerzos ayuden a crear un ambiente favorable para el desarrollo y promoción de una cultura de salud en la población escolar y sus familias.

\section{correspondencia:}

\section{Enrique Serrano}

marcos.serrano@upch.pe

\section{REFERENCIAS BIBLIOGRÁFICAS}

1. Armstrong WA, Oberg C, Orellana J. 2011. Presencia de huevos de parásitos con potencial zoonótico en parques y plazas públicas de la ciudad de Temuco, Región de La Araucanía, Chile. Archivos de medicina veterinaria, 43: 127-134.

2. Atías A. 1994. Parasitología Clínica. $3^{\text {ra }}$ Ed. Santiago de Chile. Ed. Mediterráneo. p314-318.

3. Breña JP, Huayanay L, Hernández RA, Espinoza Y, Roldán W, Maguiña C. 2007. Seroprevalencia de Toxocariosis en niños de Instituciones Educativas del distrito de San Juan de Lurigancho. Congreso Peruano de Enfermedades Infecciosas y Tropicales. Lima, Perú.

4. Chávez A, Casas E., Serrano M., Cajas J, Velarde J., La Rosa V., López J. 2002. Riesgo de contraer enfermedades parasitarias en los parques públicos de Lima y Callao. Revista de Investigaciones Veterinarias del Perú, 13(2): 84-91.

5. Delgado O, Rodríguez AJ. 2009. Aspectos clínico epidemiológicos de la toxocariasis: una enfermedad desatendida en Venezuela y América Latina. Boletín de Malariología y Salud Ambiental, 49: 1-33.

6. Espinoza Y. Roldan W, Huapaya P, Huiza A, Jimenez S, Sevilla C. 2006. Prevalencia de anticuerpos IgG anti-Toxocara, en pobladores del distrito de Perené, departamento de Junín. Anales de la Facultad de Medicina. 67(1): S66.

7. Espinoza Y. Huapaya P, Roldan W, Jiménez S, Arce Z, Lopez E. 2008. Clinical and serological evidence of Toxocara infection in school children from Morrope district, Lambayeque, Peru. Revista do Instituto de Medicina Tropical de São Paulo, 50(2): 101-105.

8. Ghiani, H. 2001. Toxocariasis y asma. Arch. alerg. inmunol. clin 32(2, supl), S102-S105.

9. Guillinta Y. 2011. Presencia de huevos de toxocara spp., en suelo de ambientes frecuentados por niños en edad escolar. Tesis de Bachiller en Medicina Veterinaria y Zootecnia. Lima: Universidad Peruana Cayetano Heredia. 32p.

10. Hendrix C; Homer S; Kellman N; Harrelson G; Bruhn 
B. 1996. Cutaneous larva migrans and enteric hookworm infections. Journal of the American Veterinary Medical Association, 209(10): 1763-1767.

11. Huapaya HP, Espinoza Y, Roldán W, Jiménez S. 2009. Toxocariosis humana: ¿problema de Salud Pública? Anales de la Facultad de Medicina 70: 283-290.

12. Leguía G. 2002. Enfermedades parasitarias de perros y gatos. 2a ed. Lima: Ed. Del Mar. 155p.

13. Mendoza D, Lozano S, Jaimes M. 2010. Exposición al parásito Toxocara canis en una población escolar de la comuna 7 del distrito de Santa Marta, Colombia. Duazary 7(2).

14. Minvielle M, Taus M, Ciarmela M, Francisconi M, Barlasina M, Pezzani B, Gasparovic A, Raffo A, Goldaracena C. 2003.Aspectos epidemiológicos asociados a toxocarosis en Gualeguaychú, Entre Ríos. Argentina. Parasitología latinoamericana, 58: 128130.

15. Miranda-Sousa A, Alzamora B, Maguiña C, Tobaru L, Yarlequé C, Terashima A, Gotuzzo E. 1999. Primer reporte en el Perú de Toxocariasis ocular: análisis de 21 casos. Bol Soc Peruana Med Interna 12: 20-8.

16. Scaini1 C, Navarrete R, Lovatel, Alix M, Anjos F, Susin L, Mendonça V. 2003. Contaminação ambiental por ovos e larvas de helmintos em fezes de cães na área central do Balneário Cassino, Rio Grande do Sul. Revista da Sociedade Brasileira de Medicina Tropical 36(5): 617-619,

17. Scaini C, Toledo R, Lovatel R, Dionello M. Gatti F, Susin L, Signorini V. 2003. Contaminação ambiental por ovos e larvas de helmintos em fezes de cães na área central do Balneário Cassino, Rio Grande do Sul. Revista da Sociedade Brasileira de Medicina Tropical 36: 617-619.

Recibido 19/06/2014

Aceptado 23/12/2014 